\title{
ОБ ОДНОМ АЛГОРИТМЕ РЕКОНСТРУКЦИИ ВОЗМУЩЕНИЯ НЕЛИНЕЙНОЙ СИСТЕМЫ
}

\section{В. И. Максимов}

\begin{abstract}
Рассматривается задача реконструкции неизвестного возмущения системы нелинейных обыкновенных дифференциальных уравнений, которая имеет две особенности. Во-первых, предполагается, что измеряются (с ошибкой) в дискретные, достаточно частые, моменты времени фазовые координаты заданной динамической системы. Во-вторых, относительно неизвестного возмущения, действующего на систему, известно лишь то, что оно является элементом пространства функций, суммируемых с квадратом евклидовой нормы, т. е. может быть неограниченным. Указанные предположения ведут к невозможности точного восстановления. Учитывая данную особенность, мы конструируем устойчивый к информационным помехам и погрешностям вычислений алгоритм решения рассматриваемой задачи, который основан на сочетании элементов теории некорректных задач с известным в теории позиционных дифференциальных игр методом экстремального сдвига.
\end{abstract}

Ключевые слова: линейные управляемые системы, динамическое восстановление.

V. I. Maksimov. On an algorithm for the reconstruction of a perturbation in a nonlinear system.

A problem of reconstruction of an unknown perturbation in a system of nonlinear ordinary differential equations is considered. The methods of solution of such problems are well known. In this paper we study a problem with two peculiarities. First, it is assumed that the phase coordinates of the dynamical system are measured (with error) at discrete sufficiently frequent times. Second, the only information known about the perturbation acting on the system is that its Euclidean norm is square integrable; i.e., the perturbation can be unbounded. Since the exact reconstruction is impossible under these assumptions, we design a solution algorithm that is stable under information noise and computation errors. The algorithm is based on the combination of elements of the theory of ill-posed problems with the extremal shift method known in the theory of positional differential games.

Keywords: linear control systems, dynamic reconstruction.

MSC: 49N45, 93B52

DOI: $10.21538 / 0134-4889-2020-26-1-156-166$

\section{1. Введение. Постановка задачи}

Рассматривается нелинейная система дифференциальных уравнений

$$
\dot{y}(t)=f(t, x(t), y(t), u(t)), \quad t \in T=[0, \vartheta],
$$

с начальным условием

$$
y(0)=y_{0} .
$$

Здесь $0<\vartheta<+\infty, x \in \mathbb{R}^{n}, y \in \mathbb{R}^{N}, u \in \mathbb{R}^{r}, f(t, x, y, u)=f_{1}(t, x, y)+B u, f_{1}$ - липшицева функция с константой Липшица $L, u$ - возмущение, $B$ - стационарная матрица соответствующей размерности, $x(\cdot) \in L_{\infty}\left(T ; \mathbb{R}^{n}\right)$ - некоторая функция. Предполагается, что на систему (1.1) действует неизвестное возмущение $u(\cdot) \in L_{2}\left(T ; \mathbb{R}^{r}\right)$. В дискретные, достаточно частые, моменты времени $\tau_{i} \in \Delta=\left\{\tau_{i}\right\}_{i=0}^{m} \quad\left(\tau_{0}=0, \tau_{m}=\vartheta, \tau_{i+1}=\tau_{i}+\delta\right)$ измеряется фазовое состояние системы $(1.1) y\left(\tau_{i}\right)=y\left(\tau_{i} ; y_{0}, x(\cdot), u(\cdot)\right)$. Состояния $y\left(\tau_{i}\right), i \in[0: m-1]$ измеряются с ошибкой. Результаты измерений - векторы $\xi_{i}^{h} \in \mathbb{R}^{N}$ - удовлетворяют неравенствам

$$
\left|y\left(\tau_{i}\right)-\xi_{i}^{h}\right|_{N} \leq h .
$$


Предполагается, что функция $x(\cdot)$ известна неточно. Именно вместо функции $x(\cdot)$ известна функция $\phi^{\nu}(\cdot) \in L_{\infty}\left(T ; \mathbb{R}^{n}\right)$ со свойством

$$
\left|x(t)-\phi^{\nu}(t)\right|_{n} \leq \nu \quad \text { при п.в. } \quad t \in T .
$$

Здесь $h \in(0,1)$ и $\nu=\nu(h)-$ уровни погрешности измерения, символ $|\cdot|_{n}$ означает евклидову норму в пространстве $\mathbb{R}^{n}$. Требуется указать алгоритм приближенного восстановления неизвестного возмущения по результатам неточных измерений $y\left(\tau_{i}\right)$. Таким образом, рассматривается задача, состоящая в построении алгоритма, который по текущим измерениям величин $y\left(\tau_{i}\right)$ в "реальном времени" формирует (по принципу обратной связи) некоторую функцию $u^{h}=u^{h}(\cdot)$, являющуюся приближением (в метрике пространства $\left.L_{2}\left(T ; \mathbb{R}^{r}\right)\right)$ некоторого возмущения, порождающего решение $y(\cdot)$ уравнения (1.1).

Сформулированная выше задача является задачей динамического восстановления (реконструкции). Можно выделить два подхода к восстановлению недоступной прямому наблюдению информации о системе. Один связан с теорией оценивания управляемых систем, нацеленной на синтез максимальной текущей информации о состояниях системы. Такая информация обычно представляется в виде так называемых информационных множеств, объединяющих все состояния системы, не противоречащие текущей истории наблюдений [1-3]. Другой подход был развит в исследованиях [4-11]. Он основан на комбинации методов теории позиционного управления [12] и некорректных задач [13;14]. Если возмущение $u(\cdot)$ стеснено мгновенными ограничениями, обсуждаемая задача может быть решена на основе конструкций работ $[5 ; 6]$.

В данной статье мы рассмотрим случай отсутствия мгновенных ограничений. Вследствие этого будем считать, что неизвестное возмущение может быть неограниченным, являясь функцией суммируемой с квадратом евклидовой нормы. Другие задачи динамического восстановления, методы решения которых основаны на соответствующих модификациях метода экстремального сдвига, обсуждались, например, в работах [7-11]. При этом в [7;8] рассматривался случай измерения "всех" координат. Случаю измерения части фазовых координат посвящены работы [9] (линейная система обыкновенных дифференциальных уравнений), [10] (система с последействием), [11] (система с распределенными параметрами). Следует отметить, что обсуждаемый в настоящей статье подход к решению задач реконструкции отличается от стандартных подходов, среди которых можно отметить, например, подходы основанные на стохастических методах [16;17], методах параметризации [17], методах двухступенчатой оптимизации [19] и другие.

\section{2. Метод решения задачи}

Перейдем к описанию метода решения рассматриваемой задачи. Как было отмечено выше, метод основан на конструкциях теории управления с обратной связью. При этом задача динамической реконструкции заменяется задачей позиционного управления некоторой подходящим образом подобранной динамической системой.

Пусть для каждого $h \in(0,1)$ фиксировано семейство $\Delta_{h}$ разбиений отрезка $T$ контрольными моментами времени $\tau_{h, i}$ :

$$
\Delta_{h}=\left\{\tau_{h, i}\right\}_{i=0}^{m_{h}}, \quad \tau_{h, 0}=0, \quad \tau_{h, m_{h}}=\vartheta, \quad \tau_{h, i+1}=\tau_{h, i}+\delta(h), \quad \delta(h) \in(0,1) .
$$

Введем вспомогательную систему, описываемую векторным дифференциальным уравнением

$$
\dot{w}^{h}(t)=f_{1}\left(\tau_{i}, \phi^{\nu}(t), \xi_{i}^{h}\right)+B u_{i}^{h} \quad \text { при п.в. } \quad t \in\left[\tau_{i}, \tau_{i+1}\right) \quad\left(i \in\left[0: m_{h}-1\right]\right)
$$

с начальным состоянием $w^{h}(0)=\xi_{0}^{h}$. Закон $U(\cdot, \cdot): \mathbb{R}^{N} \times \mathbb{R}^{N} \mapsto \mathbb{R}^{r}$ формирования управления $u^{h}(\cdot)$ этой системой конструируется таким образом, что при соответствующем согласовании ряда параметров кусочно-постоянная функция $u^{h}(\cdot)$ вида

$$
u^{h}(t)=u_{i}^{h}=U\left(\xi_{i}^{h}, w^{h}\left(\tau_{i}\right)\right) \quad \text { при п.в. } \quad \mathrm{t} \in\left[\tau_{i}, \tau_{i+1}\right) \quad\left(i \in\left[0: m_{h}-1\right]\right)
$$


аппроксимирует неизвестный вход.

Следует отметить, что одно и то же решение системы (1.1) может вызываться не единственным возмущением. Пусть $\mathcal{U}(x(\cdot), y(\cdot))$ - множество всех возмущений из $L_{2}\left(T ; \mathbb{R}^{r}\right)$, порождающих решение $y(\cdot)$ системы $(1.1)$, т. е.

$$
\mathcal{U}(x(\cdot), y(\cdot))=\left\{\tilde{u}(\cdot) \in L_{2}\left(T ; \mathbb{R}^{r}\right): \dot{y}(t)-f_{1}(t, x(t), y(t))=B \tilde{u}(t) \quad \text { при п.в. } \quad t \in T\right\} .
$$

Символом $u_{*}(\cdot)$ обозначим минимальное по $L_{2}\left(T ; \mathbb{R}^{r}\right)$-норме возмущение из $\mathcal{U}(x(\cdot), y(\cdot))$, порождающее решение $y(\cdot)$ системы (1.1), т. е.

$$
u_{*}(\cdot)=\arg \min _{u(\cdot) \in \mathcal{U}(x(\cdot), y(\cdot))}|u(\cdot)|_{L_{2}\left(T ; \mathbb{R}^{r}\right)} .
$$

Нетрудно видеть, что такое возмущение существует и единственно. Следуя принятому в теории некорректных задач подходу $[13 ; 14]$ мы будем восстанавливать $u_{*}(\cdot)$.

\section{3. Алгоритм решения}

Укажем алгоритм решения рассматриваемой задачи. Возьмем некоторые семейство $\Delta_{h}$ $(2.1)$, а также функцию $\alpha(h):(0,1) \rightarrow(0,1)$.

До начала работы алгоритма фиксируем величины $h \in(0,1), \nu=\nu(h), \alpha=\alpha(h)$ и разбиение $\Delta_{h}=\left\{\tau_{h, i}\right\}_{i=0}^{m_{h}}$ вида (2.1). Работу алгоритма разобьем на однотипные шаги. В течение $i$-го шага, осуществляемого на промежутке времени $\delta_{i}=\left[\tau_{i}, \tau_{i+1}\right), \tau_{i}=\tau_{h, i}$, выполняются следующие операции. Сначала, в момент $\tau_{i}$, вычисляется вектор $u_{i}^{h}$ по формуле $(2.3)$, в которой

$$
U\left(\xi_{i}^{h}, w^{h}\left(\tau_{i}\right)\right)=U_{\alpha}\left(\xi_{i}^{h}, w^{h}\left(\tau_{i}\right)\right)=-\alpha^{-1} B^{\prime}\left(w^{h}\left(\tau_{i}\right)-\xi_{i}^{h}\right) .
$$

Здесь штрих означает транспонирование. Затем на вход системы (2.2) подается управление $u^{h}(t)$ вида (2.3), (3.1). Под действием этого управления решение системы (2.2) переходит из состояния $w^{h}\left(\tau_{i}\right)$ в состояние $w^{h}\left(\tau_{i+1}\right)$. Работа алгоритма заканчивается в момент $\vartheta$.

Оказывается, что при определенном согласовании величин $h, \delta(h), \nu(h), \alpha(h)$ функция $u^{h}(\cdot)$ является аппроксимацией $u(\cdot)$. Прежде чем перейти к доказательству этого факта, приведем две леммы, которые понадобятся в дальнейшем.

3 а м е ч а н и е 1. Для случая, когда правая часть системы (1.1) не зависит от функции $x(\cdot)$, т. е. $f=f_{1}(t, y)+B u$, в работе [7] указан алгоритм решения рассматриваемой задачи. При этом в качестве модели бралась система

$$
\dot{w}^{h}(t)=f_{1}\left(\tau_{i}, \xi_{i}^{h}\right)+B u_{i}^{h}+v_{i}^{h}, \quad t \in \delta_{i},
$$

в которой управления $u_{i}^{h}$ и $v_{i}^{h}$ вычислялись по формулам

$$
u_{i}^{h}=\alpha(h)^{-1} B^{\prime}\left(\xi_{i}^{h}-w^{h}\left(\tau_{i}\right)\right), \quad v_{i}^{h}=c \delta(h) \alpha^{-2}(h)\left(\xi_{i}^{h}-w^{h}\left(\tau_{i}\right)\right),
$$

где $c$ - некоторая положительная константа. Там же было показано, что при соответствующем согласовании параметров $h, \alpha(h)$ и $\delta(h)$ имеет место сходимость $u^{h}(\cdot)$ к $u_{*}(\cdot)$ в $L_{2}\left(T ; \mathbb{R}^{r}\right)$. При доказательстве этой сходимости существенную роль играла функция $v^{h}(\cdot)$.

В настоящей работе, в отличие от [7], мы, во-первых, рассмотрим правую часть $f$, зависящую от некоторой функции $x(\cdot)$. Во-вторых, мы покажем, что в модели можно полагать $v^{h}(t)=0, t \in T$.

Лемма 1 [6, с. 29]. Пусть $x_{1}(\cdot) \in L_{\infty}\left(T_{*} ; \mathbb{R}^{n}\right), y_{1}(\cdot) \in W\left(T_{*} ; \mathbb{R}^{n}\right), T_{*}=[a, b],-\infty<a<$ $b<+\infty$,

$$
\left|\int_{a}^{t} x_{1}(\tau) d \tau\right|_{n} \leq \varepsilon, \quad\left|y_{1}(t)\right|_{n} \leq K \quad \forall t \in T_{*} .
$$


Тогда при всех $t \in T_{*}$ верно неравенство

$$
\left|\int_{a}^{t}\left(x_{1}(\tau), y_{1}(\tau)\right) d \tau\right| \leq \varepsilon\left(K+\operatorname{var}\left(T_{*} ; y_{1}(\cdot)\right)\right) .
$$

Здесь символ $\operatorname{var}\left(T_{*} ; y_{1}(\cdot)\right)$ означает вариацию функции $y_{1}(\cdot)$ на отрезке $T_{*}$, символ $(\cdot, \cdot)-$ скалярное произведение в соответствующем конечномерном евклидовом пространстве, символ $|\cdot|$ - модуль числа, а символ $W\left(T_{*} ; \mathbb{R}^{n}\right)$ - множество функций $y(\cdot): T_{*} \rightarrow \mathbb{R}^{n}$ с ограниченной вариацией.

Заметим, что встречающиеся в настоящей работе постоянные $C_{j}, k_{j}, k^{(j)}$ зависят от структуры системы (1.1) и не зависят от $h, \alpha, \delta, \nu$.

Лемма 2. Пусть $\alpha(h) \rightarrow 0, \delta(h) \alpha^{-2}(h) \rightarrow 0$ nри $h \rightarrow 0$. Тогда можно указать такое $h_{1} \in(0,1)$, что при всех $h \in\left(0, h_{1}\right), t \in T$ для некоторых положстельных $C_{1}-C_{3}$ справедливы неравенства

$$
\begin{gathered}
\varepsilon_{*}(t) \leq C_{1} \rho_{1}(\alpha, \delta, h, \nu), \\
\int_{0}^{\vartheta}\left|u^{h}(\tau)\right|_{r}^{2} d \tau \leq\left(1+C_{2} \alpha \delta^{-2}\right) \int_{0}^{\vartheta}|u(\tau)|_{r}^{2} d \tau+C_{3} \rho(\alpha, \delta, h, \nu) \alpha^{-1},
\end{gathered}
$$

где $\varepsilon_{*}(t)=0.5\left|w^{h}(t)-y(t)\right|_{N}^{2}, \rho_{1}(\alpha, \delta, h, \nu)=\rho(\alpha, \delta, h, \nu)+\alpha+\delta+\delta^{2} \nu^{2}, \rho(\alpha, \delta, h, \nu)=\alpha^{2} \delta+$ $h^{2} \delta^{-1}+h+\alpha^{2} \nu^{2} \delta^{-1}$.

Д о к а з а т е л ь с т в о. Рассмотрим изменение величины $\varepsilon_{*}(t)$ при $t \in T$. Для $t \in \delta_{i}=$ $\left[\tau_{i}, \tau_{i+1}\right), i \in[0: m-1]$ имеем

$$
\varepsilon_{*}(t)=0.5\left|w^{h}\left(\tau_{i}\right)-y\left(\tau_{i}\right)+\int_{\tau_{i}}^{t}\left(f^{i}(\tau)+B^{i}(\tau)\right) d \tau\right|_{N}^{2},
$$

где $m=m_{h}, \tau_{i}=\tau_{h, i}$,

$$
f^{i}(t)=f_{1}\left(\tau_{i}, \phi^{\nu}(t), \xi_{i}^{h}\right)-f_{1}(t, x(t), y(t)), \quad B^{i}(t)=B\left(u_{i}^{h}-u(t)\right) \quad \text { при п.в. } \quad \mathrm{t} \in \delta_{i} .
$$

В таком случае при $t \in \delta_{i}$ справедливо равенство

$$
\varepsilon_{*}(t)=\varepsilon_{*}\left(\tau_{i}\right)+\sum_{j=1}^{5} \nu_{i}^{(j)}(t) .
$$

Здесь

$$
\begin{aligned}
& \nu_{i}^{(1)}(t)=\left(w^{h}\left(\tau_{i}\right)-y\left(\tau_{i}\right), \int_{\tau_{i}}^{t} f^{i}(\tau) d \tau\right), \quad \nu_{i}^{(2)}(t)=0.5\left|\int_{\tau_{i}}^{t} f^{i}(\tau) d \tau\right|_{N}^{2}, \\
& \nu_{i}^{(3)}(t)=\left(w^{h}\left(\tau_{i}\right)-y\left(\tau_{i}\right), \int_{\tau_{i}}^{t} B^{i}(\tau) d \tau\right), \\
& \nu_{i}^{(4)}(t)=\left(\int_{\tau_{i}}^{t} f^{i}(\tau) d \tau, \int_{\tau_{i}}^{t} B^{i}(\tau) d \tau\right), \quad \nu_{i}^{(5)}(t)=0.5\left|\int_{\tau_{i}}^{t} B^{i}(\tau) d \tau\right|_{N}^{2}, \quad t \in \delta_{i} .
\end{aligned}
$$

Всюду в доказательстве этой леммы $\alpha=\alpha(h), \nu=\nu(h), \delta=\delta(h)$. Нетрудно видеть, что при всех $i$ верны неравенства (см. (3.1))

$$
\left|u_{i}^{h}\right|_{r}=\left|\frac{B^{\prime}\left(\xi_{i}^{h}-w^{h}\left(\tau_{i}\right)\right)}{\alpha}\right|_{r} \leq \frac{b_{*}}{\alpha} Q_{i},
$$


где $Q_{i}=h+\left(2 \varepsilon_{*}\left(\tau_{i}\right)\right)^{1 / 2}, b_{*}-$ евклидова норма матрицы $B$. Заметим, что при $t \in\left[\tau_{i}, \tau_{i+1}\right]$ верна оценка

$$
\left|f^{i}(t)\right|_{N} \leq L\left(\delta+h+\nu+\left|y(t)-y\left(\tau_{i}\right)\right|_{N}\right) \leq L Q_{t}^{(i)} .
$$

Здесь

$$
Q_{t}^{(i)}=\delta+h+\nu+\int_{\tau_{i}}^{t}|\dot{y}(\tau)|_{N} d \tau
$$

В свою очередь из соотношения (3.5) следует неравенство

$$
\nu_{i}^{(1)}(t) \leq L\left(2 \varepsilon_{*}\left(\tau_{i}\right)\right)^{1 / 2} \delta Q_{t}^{(i)} \leq \frac{\delta^{2}}{4 \alpha^{2}} \varepsilon_{*}\left(\tau_{i}\right)+k_{1} \alpha^{2}\left(Q_{t}^{(i)}\right)^{2}, \quad t \in \delta_{i} .
$$

Обозначим $U_{i p}(t)=\int_{\tau_{i}}^{t}|u(\tau)|_{r}^{p} d \tau, p=1,2$. Имеем

$$
\nu_{i}^{(2)}(t) \leq k_{2} \delta^{2}\left(Q_{t}^{(i)}\right)^{2}, \quad t \in \delta_{i} .
$$

Также верно неравенство (см. (3.4))

$$
\left|\int_{\tau_{i}}^{t} B^{i}(\tau) d \tau\right|_{N} \leq b_{*}\left(U_{i 1}(t)+\delta\left|u_{i}^{h}\right|_{N}\right) \leq b_{*}\left(b_{*} \delta \alpha^{-1} Q_{i}+U_{i 1}(t)\right), \quad t \in \delta_{i} .
$$

В силу (3.4) и (3.8) имеет место соотношение

$$
\begin{gathered}
\nu_{i}^{(3)}(t) \leq \int_{\tau_{i}}^{t}\left(w^{h}\left(\tau_{i}\right)-\xi_{i}^{h}, B^{i}(\tau)\right) d \tau+h b_{*}\left\{b_{*} \delta \alpha^{-1}\left(h+\left(2 \varepsilon_{*}\left(\tau_{i}\right)\right)^{1 / 2}\right)+U_{i 1}(t)\right\} \\
\quad \leq \int_{\tau_{i}}^{t}\left(w^{h}\left(\tau_{i}\right)-\xi_{i}^{h}, B^{i}(\tau)\right) d \tau+k_{3}\left\{\left(1+\frac{\delta}{\alpha}\right) h^{2}+h U_{i 1}(t)\right\}+\frac{\delta^{2}}{4 \alpha^{2}} \varepsilon_{*}\left(\tau_{i}\right) .
\end{gathered}
$$

Нетрудно видеть, что при $t \in\left[\tau_{i}, \tau_{i+1}\right]$ верны неравенства

$$
\begin{gathered}
\nu_{i}^{(4)}(t) \leq k_{4}\left\{\frac{\delta^{2}}{\alpha} Q_{t}^{(i)}\left(h+\varepsilon_{*}^{1 / 2}\left(\tau_{i}\right)\right)+\delta Q_{t}^{(i)} U_{i 1}(t)\right\} \\
\leq \frac{\delta^{2}}{4 \alpha^{2}} \varepsilon_{*}\left(\tau_{i}\right)+k_{5}\left\{\delta^{2}\left(Q_{t}^{(i)}\right)^{2}+\frac{\delta^{2}}{\alpha^{2}} h^{2}+\delta U_{i 2}(t)\right\}, \\
\nu_{i}^{(5)}(t) \leq 0.5 b_{*}^{2}\left(U_{i 1}(t)+\delta b_{*} \frac{Q_{i}}{\alpha}\right)^{2} \leq 4 b_{*}^{4} \frac{\delta^{2}}{\alpha^{2}} \varepsilon_{*}\left(\tau_{i}\right)+k_{6}\left\{h^{2} \frac{\delta^{2}}{\alpha^{2}}+\delta U_{i 2}(t)\right\} .
\end{gathered}
$$

Объединяя соотношения (3.6), (3.7), (3.9)-(3.11) и учитывая условие $\delta(h) \alpha^{-2}(h) \rightarrow 0$ при $h \rightarrow 0$, при $t \in\left[\tau_{i}, \tau_{i+1}\right]$ получаем

$$
\varepsilon_{*}(t) \leq \varepsilon_{*}\left(\tau_{i}\right)+\left(1+4 b_{*}^{4} \frac{\delta^{2}}{\alpha^{2}}\right) \varepsilon_{*}\left(\tau_{i}\right)+k_{7}\left\{\delta U_{i 2}(t)+\left(\alpha^{2}+\delta^{2}\right)\left(Q_{t}^{(i)}\right)^{2}+h^{2}+h U_{i 1}(t)\right\} .
$$

Далее, имеем

$$
\left(\alpha^{2}+\delta^{2}\right) \sum_{i=0}^{m-1}\left(Q_{\tau_{i+1}}^{(i)}\right)^{2} \leq k_{8} \alpha^{2} \sum_{i=0}^{m-1}\left(h^{2}+\delta^{2}+\nu^{2}+\delta Q_{i, \tau_{i+1}}\right) \leq k_{9}\left\{\alpha^{2} \delta+\alpha^{2}\left(h^{2}+\nu^{2}\right) \delta^{-1}\right\} .
$$


Здесь $Q_{i, t}=\int_{\tau_{i}}^{t}|\dot{y}(\tau)|_{N}^{2} d \tau, t \in\left[\tau_{i}, \tau_{i+1}\right]$. Введем величину

$$
\mu(t)=2 \varepsilon_{*}(t)+\alpha \int_{0}^{t}\left\{\left|u^{h}(\tau)\right|_{r}^{2}-|u(\tau)|_{r}^{2}\right\} d \tau
$$

Воспользовавшись правилом определения управления $u^{h}(\cdot)$, из (3.12) получаем

$$
\mu(t) \leq \mu\left(\tau_{i}\right)+1\left(1+4 b_{*}^{4} \frac{\delta^{2}}{\alpha^{2}}\right) \varepsilon_{*}\left(\tau_{i}\right)+k_{7}\left\{\delta U_{i 2}(t)+\left(\alpha^{2}+\delta^{2}\right)\left(Q_{t}^{(i)}\right)^{2}+h^{2}+h U_{i 1}(t)\right\} .
$$

Пусть

$$
\gamma_{*}(t)=2 \varepsilon_{*}(t)+\alpha \int_{0}^{t}\left|u^{h}(\tau)\right|_{r}^{2} d \tau
$$

Из (3.14), учитывая неравенство $\left(1+\delta \alpha^{-1}\right) \delta \alpha^{-1} \leq$ const, выводим при $t \in\left[\tau_{i}, \tau_{i+1}\right]$ оценку

$$
\gamma_{*}(t) \leq\left(1+4 b_{*}^{4} \frac{\delta^{2}}{\alpha^{2}}\right) \gamma_{*}\left(\tau_{i}\right)+\left(\alpha+k_{10} \delta\right) U_{i 2}(t)+k_{11}\left\{\left(\alpha^{2}+\delta^{2}\right)\left(Q_{t}^{(i)}\right)^{2}+h^{2}+h U_{i 1}(t)\right\} .
$$

В силу (3.13) из (3.15) стандартным образом (см., например, [12]) следует

$$
\gamma_{*}\left(\tau_{i+1}\right) \leq\left[\gamma_{*}(0)+\left(\alpha+k_{10} \delta\right) U^{(i+1)}+k_{12} \rho\right] \exp \left\{4 b_{*}^{4} \frac{\delta}{\alpha^{2}} \tau_{i+1}\right\}, \quad i \in[0: m-1] .
$$

Здесь $\rho=\rho(\alpha, \delta, h, \nu), U^{(i+1)}=\int_{0}^{\tau_{i+1}}|u(\tau)|_{r}^{2} d \tau$. Далее, из последнего неравенства, учитывая тот факт, что $\gamma_{*}(0) \leq h^{2}$ (см. (1.2) при $i=0$ ), получаем

$$
\gamma_{*}\left(\tau_{i}\right) \leq\left[\left(k_{13} \rho+\left(\alpha+k_{10} \delta\right) U^{(i)}\right] \exp \left\{\frac{4 b_{*}^{4} \delta}{\alpha^{2}} \tau_{i}\right\} .\right.
$$

В силу условия $\delta(h) \alpha^{-2}(h) \rightarrow 0$ при $h \rightarrow 0$ найдется такое число $h^{*} \in(0,1)$, что при всех $h \in\left(0, h^{*}\right)$ справедливо неравенство

$$
\exp \left\{4 b_{*}^{4} \vartheta \delta \alpha^{-2}\right\} \leq 1+k_{14} \delta \alpha^{-2} .
$$

В таком случае, учитывая (3.17), из (3.16) выводим оценку, справедливую при всех $h \in\left(0, h^{*}\right)$, $i \in[0: m]$

$$
2 \varepsilon_{*}\left(\tau_{i}\right) \leq \gamma_{*}\left(\tau_{i}\right) \leq k_{15} \rho+\left(\alpha+k_{10} \delta\right)\left(1+k_{14} \delta \alpha^{-2}\right) U^{(i)} .
$$

В свою очередь, из (3.18) имеем

$$
\begin{aligned}
& \int_{0}^{\tau_{i}}\left|u^{h}(\tau)\right|_{r}^{2} d \tau \leq\left(1+k_{10} \delta \alpha^{-1}\right)\left(1+k_{14} \delta \alpha^{-2}\right) U^{(i)}+k_{15} \rho \alpha^{-1} \\
& \leq\left(1+k_{16} \frac{\delta}{\alpha^{2}}\right) U^{(i)}+k_{15} \rho \alpha^{-1}, \quad i \in[0: m], \quad h \in\left(0, h^{*}\right) .
\end{aligned}
$$

Положив $i=m$, получим неравенство (3.3). Проверим неравенство (3.2). При $t \in\left[\tau_{i}, \tau_{i+1}\right]$ верна оценка

$$
\left(2 \varepsilon_{*}(t)\right)^{1 / 2} \leq\left(2 \varepsilon_{*}\left(\tau_{i}\right)\right)^{1 / 2}+I_{t, i}+\left|\int_{\tau_{i}}^{t} B\left\{u_{i}^{h}-u(\tau)\right\} d \tau\right|_{N}
$$


где (см. (3.5))

$$
I_{t, i}=\int_{\tau_{i}}^{t}\left|f_{1}(\tau, x(\tau), y(\tau))-f_{1}\left(\tau_{i}, \phi^{\nu}(\tau), \xi_{i}^{h}\right)\right|_{n} d \tau \leq \delta L\left(h+\delta+\nu+\int_{\tau_{i}}^{t}|\dot{y}(\tau)|_{N} d \tau\right) .
$$

Поэтому из (3.20), воспользовавшись неравенством (3.21), а также неравенством

$$
\max _{i \in[0: m-1]} \int_{\tau_{i}}^{\tau_{i+1}}|B u(t)|_{N} d t \leq k_{17} \delta^{1 / 2}
$$

получаем $\left(2 \varepsilon_{*}(t)\right)^{1 / 2} \leq\left(2 \varepsilon_{*}\left(\tau_{i}\right)\right)^{1 / 2}+k_{18}\left\{\delta \nu+\delta\left|u_{i}^{h}\right|_{r}+\delta^{1 / 2}\right\}$. Таким образом,

$$
2 \varepsilon_{*}(t) \leq k_{19}\left\{\varepsilon_{*}\left(\tau_{i}\right)+\delta+\delta^{2}\left|u_{i}^{h}\right|_{r}^{2}+\delta^{2} \nu^{2}\right\}, \quad t \in\left[\tau_{i}, \tau_{i+1}\right] .
$$

Далее, в силу (3.4) имеем

$$
\delta^{2}\left|u_{i}^{h}\right|_{r}^{2} \leq 2 b_{*}^{2} \delta^{2} \alpha^{-2}\left(h^{2}+2 \varepsilon_{*}\left(\tau_{i}\right)\right) \leq k_{20}\left(h^{2}+\varepsilon_{*}\left(\tau_{i}\right)\right) .
$$

Значит, ввиду (3.22), (3.23) при $t \in\left[\tau_{i}, \tau_{i+1}\right]$ справедливо неравенство

$$
2 \varepsilon_{*}(t) \leq k_{21}\left\{\varepsilon_{*}\left(\tau_{i}\right)+\delta+\delta^{2} \nu^{2}\right\}
$$

Отсюда и из (3.18) получаем

$$
2 \varepsilon_{*}(t) \leq k_{22}\left(\rho+\delta+\delta^{2} \nu^{2}+\left(\alpha+k_{10} \delta\right)\left(1+k_{14} \delta \alpha^{-2}\right) \int_{0}^{t}|u(\tau)|_{r}^{2} d \tau\right) \leq k_{23} \rho_{1}(\alpha, \delta, h, \nu), \quad t \in T .
$$

Неравенство (3.2) следует из последнего неравенства. Лемма доказана.

С помощью леммы 2 стандартным образом (см., например, [6]) может быть доказана

Теорема 1. Пусть выполнены условия леммы 2. Пусть также

$$
\begin{gathered}
\rho_{1}(\alpha(h), \delta(h), h, \nu(h)) \rightarrow 0, \quad \nu(h) \rightarrow 0, \\
\rho(\alpha(h), \delta(h), h, \nu(h)) \alpha^{-1}(h) \rightarrow 0 \quad \text { npu } h \rightarrow 0 .
\end{gathered}
$$

Тогда имеет место сходимость $u^{h}(\cdot) \rightarrow u_{*}(\cdot) \quad n p u \quad h \rightarrow 0$.

\section{4. Оценка скорости сходимости алгоритма}

При некоторых дополнительных условиях может быть выписана оценка скорости сходимости алгоритма.

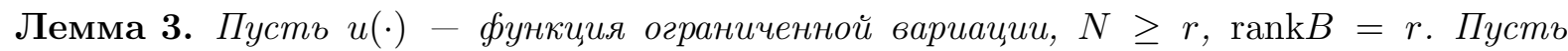
также выполнены условия леммы 2. Тогда можно указать константу $C_{4}>0$ такую, ито при всех $h \in\left(0, h_{1}\right)$ верно неравенство

$$
\int_{0}^{\vartheta}\left|u^{h}(\tau)-u(\tau)\right|_{r}^{2} d \tau \leq C_{4} \rho_{0}(\alpha, \delta, h, \nu)+C_{3} \rho(\alpha, \delta, h, \nu) \alpha^{-1} .
$$

где $\rho_{0}(\alpha, \delta, h, \nu)=\alpha^{1 / 2}+\delta^{1 / 2}+h \delta^{-1 / 2}+\alpha \nu \delta^{-1 / 4}+\nu \delta$, константа $C_{3}$ та же, что и в (3.3). 
Д о к а з а т е л ь с т в о. Учитывая липшицевость функции $f_{1}$, а также лемму 2 , заключаем, что для любых $t_{1}, t_{2} \in T, t_{1}<t_{2}$ справедливо неравенство

$$
\begin{gathered}
\left|\int_{t_{1}}^{t_{2}} B\left(u^{h}(t)-u(t)\right) d t\right|_{N}=\left|\int_{t_{1}}^{t_{2}}\left[\dot{w}^{h}(\tau)-\dot{y}(\tau)-f_{1}\left(\tau, \phi^{\nu}(\tau), \xi^{h}(\tau)\right)+f_{1}(\tau, x(\tau), y(\tau))\right] d \tau\right|_{N} \\
\leq\left|\mu_{h}\left(t_{2}\right)-\mu_{h}\left(t_{1}\right)\right|_{N}+k^{(1)}\left(\int_{t_{1}}^{t_{2}}\left[\left|\xi^{h}(\tau)-y(\tau)\right|_{N}+\left|\phi^{\nu}(\tau)-x(\tau)\right|_{n}\right] d \tau+\delta\right) \\
\leq\left|\mu_{h}\left(t_{2}\right)-\mu_{h}\left(t_{1}\right)\right|_{N}+k^{(2)} \int_{t_{1}}^{t_{2}}\left(\left|\mu_{h}(\tau)\right|_{N}+h+\delta+\nu\right) d \tau,
\end{gathered}
$$

где $\mu_{h}(t)=w^{h}(t)-y(t), \xi^{h}(\tau)=\xi_{i}^{h}$ при п.в. $\tau \in\left[\tau_{i}, \tau_{i+1}\right)$. Кроме того, в силу леммы 3 (см., (3.2)) имеем $\left|\mu_{h}(t)\right|_{N}=\left(2 \varepsilon_{*}(t)\right)^{1 / 2} \leq C_{1}^{1 / 2} \rho_{1}^{1 / 2}(\alpha, \delta, h, \nu)$. Отсюда выводим

$$
\begin{gathered}
\left|\int_{t_{1}}^{t_{2}}\left(u^{h}(t)-u(t)\right) d t\right|_{r} \leq k^{(3)}\left|\int_{t_{1}}^{t_{2}} B\left(u^{h}(t)-u(t)\right) d t\right|_{N} \leq k^{(4)}\left\{\rho_{1}^{1 / 2}(\alpha, \delta, h, \nu)+h+\delta+\nu\right\} \\
\leq k^{(5)} \rho_{0}(\alpha, \delta, h, \nu) .
\end{gathered}
$$

Снова воспользовавшись леммой 2 (см. (3.3)), получаем

$$
\begin{gathered}
\int_{0}^{\vartheta}\left|u^{h}(\tau)-u(\tau)\right|_{r}^{2} d \tau=\int_{0}^{\vartheta}\left|u^{h}(\tau)\right|_{r}^{2} d \tau-2 \int_{0}^{\vartheta}\left(u^{h}(\tau), u(\tau)\right) d \tau+\int_{0}^{\vartheta}|u(\tau)|_{r}^{2} d \tau \\
\leq\left(2+C_{2} \alpha \delta^{-2}\right) \int_{0}^{\vartheta}|u(\tau)|_{r}^{2} d \tau-2 \int_{0}^{\vartheta}\left(u^{h}(\tau), u(\tau)\right) d \tau+C_{3} \rho(\alpha, \delta, h, \nu) \alpha^{-1} \\
=2 \int_{0}^{\vartheta}\left(u(\tau)-u^{h}(\tau), u(\tau)\right) d \tau+C_{2} \alpha \delta^{-2} \int_{0}^{\vartheta}|u(\tau)|_{r}^{2} d \tau+C_{3} \rho(\alpha, \delta, h, \nu) \alpha^{-1}, \quad t \in T .
\end{gathered}
$$

В силу леммы 1 , учитывая (4.1), имеем $\sup _{t \in T}\left|\int_{0}^{t}\left(u(\tau)-u^{h}(\tau), u(\tau)\right) d \tau\right| \leq k^{(6)} \rho_{0}(\alpha, \delta, h, \nu)$. Таким образом, при всех $h \in\left(0, h_{1}\right), t \in T$ верно неравенство

$$
\int_{0}^{\vartheta}\left|u^{h}(\tau)-u(\tau)\right|_{r}^{2} d \tau \leq 2 k^{(6)} \rho_{0}(\alpha, \delta, h, \nu)+C_{3} \rho(\alpha, \delta, h, \nu) \alpha^{-1} .
$$

Отсюда следует утверждение леммы. Лемма доказана.

Нетрудно проверить, что справедлива следующая лемма.

Лемма 4. Пусть выполнены условия леммы 2. Пусть такље $\chi \in(0,1 / 2)$. Если $\delta(h)=$ $h, \alpha(h)=h^{1 / 2-\chi}(h)$, то можно указать такое число $h_{2} \in\left(0, h_{1}\right)$, что при всех $h \in\left(0, h_{2}\right)$ для некоторых положительных $C_{5}-C_{7}$ справедливы неравенства

$$
\begin{gathered}
\rho(\alpha(h), \delta(h), h, \nu(h)) \alpha^{-1}(h) \leq C_{5} h^{1 / 2-\chi}, \\
\rho_{1}(\alpha(h), \delta(h), h, \nu(h)) \leq C_{6} h^{1 / 2-\chi}, \quad \rho_{0}(\alpha(h), \delta(h), h, \nu(h)) \leq C_{7}^{1 / 4-\chi / 2} .
\end{gathered}
$$


В таком случае из лемм 2 и 4 вытекает

Следствие. Пусть выполнены условия леммы 4. Тогда существует $C_{8}>0$ такое, что имеет место неравенство

$$
\int_{0}^{\vartheta}\left|u^{h}(t)-u(t)\right|_{r}^{2} d t \leq C_{8} h^{1 / 4-\chi / 2} .
$$

\section{5. Пример}

Пусть в системе $(1.1) x(t)=\dot{z}(t)$. При этом функция $z(\cdot)$ неизвестна. Известны лишь ее свойства: $\dot{z}(0)=0, \ddot{z}(\cdot) \in L_{2}\left(T ; \mathbb{R}^{n}\right)$. В моменты $\tau_{i}$ измеряются ее значения с ошибкой $h$, т. е. вычисляются векторы $\psi_{i}^{h} \in \mathbb{R}^{n}$ такие, что $\left|\psi_{i}^{h}-z\left(\tau_{i}\right)\right|_{n} \leq h$. Задача состоит в восстановлении возмущения $u(\cdot)$, порождающего решение $y(\cdot)$ системы $(1.1)$ по измерениям $z\left(\tau_{i}\right)$ и $y\left(\tau_{i}\right)$.

Для решения задачи можно воспользоваться описанным выше алгоритмом, восстанавливая $u_{*}(\cdot)$ - минимальный в $L_{2}\left(T ; \mathbb{R}^{r}\right)$ элемент множества

$$
U(y(\cdot), z(\cdot))=\left\{u(\cdot) \in L_{2}\left(T ; \mathbb{R}^{r}\right): \dot{y}(t)=f_{1}(t, y(t), \dot{z}(t))+B u(t) \text { при п.в. } t \in T\right\} .
$$

Вместе с функцией $\alpha=\alpha(h)$ фиксируем функцию $\alpha_{1}=\alpha_{1}(h):(0,1) \rightarrow(0,1)$. Наряду с моделью (2.2) введем еще одну вспомогательную систему следующего вида:

$$
\dot{w}_{1}^{h}(t)=u_{1}^{h}(t), \quad t \in T .
$$

Начальное состояние этой системы $w_{1}^{h}(0)=0$. Управление $u_{1}^{h}(\cdot)$ будем вычислять по правилу

$$
u_{1}^{h}(t)=u_{i}^{h}=-\frac{w_{1}^{h}\left(\tau_{i}\right)-\psi_{i}^{h}}{\alpha_{1}(h)}, \quad t \in\left[\tau_{i}, \tau_{i+1}\right) .
$$

Из результатов [15] (см. теорему 5) следует справедливость неравенства

$$
\sup _{t \in T}\left|u_{1}^{h}(t)-\dot{z}(t)\right|_{n} \leq \nu(h),
$$

где $\nu(h)=C_{9}\left\{\alpha_{1}(h)+(h+\delta(h)) \alpha_{1}^{-1}(h)\right\}$. В таком случае можно считать $(\nu=\nu(h)) \phi^{\nu}(t)=$ $u_{1}^{h}(t), t \in T$. Тогда в силу теоремы 1 , если выполнены следующие условия согласования параметров

$$
\begin{gathered}
\alpha(h) \rightarrow 0, \quad \alpha_{1}(h) \rightarrow 0, \quad h^{2} \delta^{-1}(h) \rightarrow 0, \quad \alpha^{2}(h) \alpha_{1}^{2}(h) \delta^{-1}(h) \rightarrow 0, \\
\alpha^{2}(h)\left(h^{2}+\delta^{2}(h)\right) \alpha_{1}^{-1}(h) \rightarrow 0 \quad \text { при } \quad h \rightarrow 0,
\end{gathered}
$$

имеет место сходимость $u^{h}(\cdot) \rightarrow u_{*}(\cdot)$ в $L_{2}\left(T ; \mathbb{R}^{r}\right)$ при $h \rightarrow 0$. В свою очередь, соотношения (5.2) выполнены, например, если

$$
\delta(h)=C_{10} h, \quad \alpha(h)=C_{11} h^{\mu} \quad(\mu=\text { const } \in(0,1)), \quad \alpha_{1}(h)=C_{12} h^{1 / 4} .
$$

При этом $\nu(h) \leq C_{13} h^{1 / 2}, \alpha^{2}(h) \nu^{2}(h) \delta(h) \leq C_{14} h^{2 \mu}$. Заметим, что в данном примере величина $z(t)$ измеряется в дискретные моменты времени. Однако, роль $\phi^{\nu}(\cdot)$ играет функция $u_{1}^{h}(\cdot)$, которая по величинам $\psi_{i}^{h}$ определяется для всех $t \in T$.

\section{СПИСОК ЛИТЕРАТУРЫ}

1. Куржанский А.Б. Управление и наблюдение в условиях неопределенности. М.: Наука, 1977. $392 \mathrm{c.}$

2. Kurzhanski A., Valyi I. Ellipsoidal calculus for estimation and control. Boston: Birkhäuser, 1996. $284 \mathrm{p}$. 
3. Ананьев Б.И., Гусев М.И., Филиппова Т.Ф. Управление и оценивание состояний динамических систем с неопределенностью. Новосибирск: Изд-во СО РАН. 2018. 193 с.

4. Осипов Ю.С., Кряжимский А.В., Максимов В.И. Некоторые алгоритмы динамического восстановления входов // Тр. Ин-та математики и механики УрО РАН. 2011. Т. 17, № 1. С. 29-161.

5. Osipov Yu.S., Kryazhimskii A.V. Inverse problems for ordinary differential equations: dynamical solutions. Basel: Gordon and Breach, 1995. 625 p.

6. Осипов Ю.С., Кряжимский А.В., Максимов В.И. Методы динамического восстановления входов управляемых систем / УрО РАН. Екатеринбург, 2011. 291 с.

7. Максимов В.И., Пандолфи Л. О реконструкции неограниченных управлений в нелинейных динамических системах // Прикл. математика и механика. 2001. Т. 65, № 4. С. 385-390.

8. Близорукова М.С., Максимов В.И. Об одном алгоритме динамического восстановления входного воздействия // Дифференц. уравнения. 2013. Т. 49, № 1. С. 88-100.

9. Maksimov V.I. On dynamical reconstruction of an input in a linear system under measuring a part of coordinates // J. Inv. Ill-Posed Problems. 2018. Vol. 26, no. 3. P. 395-410.

10. Близорукова М.С., Максимов В.И. Об одном алгоритме реконструкции траектории и управления в системе с запаздыванием // Тр. Ин-та математики и механики УрО РАН. 2012. Т. 18 , № 1. C. $109-122$.

11. Каппель Ф., Кряжимский А.В., Максимов В.И. Динамическая реконструкция состояний и гарантирующее управление системой реакции-диффузии // Докл. АН. 2000. Т. 370 , № 5. C. 599-601.

12. Красовский Н.Н., Субботин А.И. Позиционные дифференциальные игры. М.: Наука, 1974. $456 \mathrm{c.}$

13. Тихонов А.Н., Арсенин В.Я. Методы решения некорректных задач. М.: Наука, 1978. 285 с.

14. Иванов В.K., Васин В.В., Танана В.П. Теория линейных некорректных задач и ее приложения. М.: Наука, 1978. 206 с.

15. Максимов В.И. О вычислении производной функции заданной неточно с помощью законов обратной связи // Тр. МИРАН. 2015. Т. 291. С. 231-243.

16. Fang H., Shi Y., Yu J. On stable simultaneous input and state estimation for discrete-time linear systems // Internat. J. Adaptiv. Contr. Signal Proc. 2011. Vol. 25, no. 8. P. 671-686.

17. Keller J.Y., Chabir K., Sauter D. Input reconstruction for networked control systems subject to deception attacks and data losses on control signals // Int. J. Syst. Sci. 2016. Vol. 47, no 4. P. 814-820.

18. Keller J.Y., Sauter D. Kalman filter for discrete-time stochastic linear systems subject to intermittent unknown inputs // IEEE Trans. Autom. Contr. 2013. Vol. 58, no. 7. P. 1882-1887.

19. Chabir K., Sid M.A., and Sauter D. Fault diagnosis in a networked control system under communication constraints: A quadrotor applications // Int. J. Apll. Math. Comput. Sci. 2014. Vol. 24. no. 4. P. 809-820.

Поступила 5.10.2019

После доработки 13.01.2020

Принята к публикации 20.01.2020

Максимов Вячеслав Иванович

д-р физ.-мат. наук, профессор

зав. отделом

Институт математики и механики им. Н. Н. Красовского УрО РАН;

Уральский федеральный университет

г. Екатеринбург

e-mail: maksimov@imm.uran.ru

\section{REFERENCES}

1. Kurzhanski A.B. Upravlenie i nablyudenie v usloviyakh neopredelennosti [Control and observation under the conditions of uncertainty]. Moscow: Nauka Publ., 1977, 392 p.

2. Kurzhanski A.B., Valyi I. Ellipsoidal calculus for estimation and control. Basel: Birkhäuser, 1997,321 p. ISBN: 978-0-8176-3699-9 . 
3. Ananyev B.I., Gusev M.I., Filippova T.F. Upravlenie i otsenivanie sostoyanii dinamicheskikh sistem s neopredelennost'yu [Control and estimation of dynamical systems states with uncertainty]. Novosibirsk: Siberian Branch of RAS Publ., 2018, 193 p.

4. Osipov Yu.S., Kryazhimskii A.V., Maksimov V.I. Some algorithms for the dynamic reconstruction of inputs. Proc. Steklov Inst. Math., 2011, vol. 275, suppl. 1, pp. 86-120. doi: 10.1134/S0081543811090082 .

5. Osipov Yu.S., Kryazhimskii A.V. Inverse problems for ordinary differential equations: dynamical solutions. Basel: Gordon and Breach, 1995, 625 p. ISBN: 2881249442.

6. Osipov Yu.S., Kryazhimskii A.V., and Maksimov V.I. Metody dinamicheskogo vosstanovleniya vkhodov upravlyaemykh sistem [Methods for dynamic reconstruction of inputs of control systems]. Ekaterinburg: Ural Branch of RAS Publ., 2011, 291 p.

7. Maksimov V.I., Pandolfi L. The reconstruction of unbounded controls in nonlinear dynamical systems. J. Appl. Math. Mech., 2001, vol. 65, no. 3, pp. 371-376.

8. Blizorukova M.S., Maksimov V.I. On an algorithm for dynamic reconstruction of the input. Diff. Eq., 2013, vol. 49, no. 1, pp. 88-100. doi: 10.1134/S0012266113010096.

9. Maksimov V.I. On dynamical reconstruction of an input in a linear system under measuring a part of coordinates. J. Inverse and Ill-Posed Problems, 2018, vol. 26, no. 3, pp. 395-410. doi: 10.1515 /jiip-2017-0118.

10. Blizorukova M.S., Maksimov V.I. On a reconstruction algorithm for the trajectory and control in a delay system. Proc. Steklov Inst. Math.., 2013, vol. 280, no. 1, pp. 66-79. doi: 10.1134/S0081543813020065.

11. Kappel F., Kryazhimskii A.V., Maksimov V.I. Dynamic reconstruction of states, and the guaranteeing control of a reaction-diffusion system. Dokl. Math., 2000, vol. 61, no. 1, pp. 143-145.

12. Krasovskii N.N., Subbotin A.I. Game-theoretical control problems. N Y: Springer, 1988,517 p. ISBN: 978-1-4612-8318-8. Original Russian text published in Krasovskii N.N., Subbotin A.I. Pozitsionnye differentsial'nye igry. Moscow: Nauka Publ., 1974, 456 p.

13. Tikhonov A.N., Arsenin V.Ya. Methods for Solutions of Ill-Posed Problems. N Y: Wiley, 1977,258 p. ISBN: 0470991240. Original Russian text (2nd ed.) published in Tikhonov A.N., Arsenin V.Ya. Metody resheniya nekorrektnykh zadach. Moscow: Nauka Publ., 1978, 285 p.

14. Ivanov V.K., Vasin V.V., Tanana V.P. Theory of linear ill-posed problems and its applications. Inverse and Ill-Posed Problems Series. Utrecht: VSP, 2002, 281 p. ISBN: 90-6764-367-X/hbk . Original Russian text published in Ivanov V.K., Vasin V.V., Tanana V.P. Teoriya lineinykh nekorrektnykh zadach $i$ ee prilozheniya. Moscow: Nauka Publ., 1978, 206 p.

15. Maksimov V.I. Calculation of the derivative of an inaccurately defined function by means of feedback laws. Proc. Steklov Inst. Math., 2015, vol. 291, pp. 219-231. doi: 10.1134/S0081543815080179.

16. Fang H., Shi Y., Yu J. On stable simultaneous input and state estimation for discrete-time linear systems. Internat. J. Adaptiv. Contr. Signal Proc. 2011, vol. 25, no. 8, no. 671-686.

17. Keller J.Y., Chabir K., Sauter D. Input reconstruction for networked control systems subject to deception attacks and data losses on control signals. Int. J. Syst. Sci., 2016, vol. 47, no. 4, pp. 814-820.

18. Keller J.Y., Sauter D. Kalman filter for discrete-time stochastic linear systems subject to intermittent unknown inputs. IEEE Trans. Autom. Contr., 2013, vol. 58, no. 7, pp.1882-1887.

19. Chabir K., Sid M.A., and Sauter D. Fault diagnosis in a networked control system under communication constraints: A quadrotor applications. Int. J. Apll. Math. Comput. Sci., 2014, vol. 24, no. 4, pp. 809-820.

Received November 5, 2019

Revised January 13, 2020

Accepted January 20, 2020

Vyacheslav Ivanovich Maksimov Dr. Phys.-Math. Sci., Prof., Krasovskii Institute of Mathematics and Mechanics of the Ural Branch of the Russian Academy of Sciences, Yekaterinburg, 620108 Russia; Ural Federal University, Yekaterinburg, 620083 Russia, e-mail: maksimov@imm.uran.ru .

Cite this article as: V.I.Maksimov. On an algorithm for the reconstruction of a perturbation in a nonlinear system, Trudy Instituta Matematiki i Mekhaniki URO RAN, 2020, vol. 26, no. 1, pp. 156-166. 